\title{
The COVID-19 Pandemic in Nijmegen, the Netherlands: Changes in Presented Health Problems and Demand for Primary Care
}

Henk Schers, $M D, P b D$

Chris van Weel, $M D, P b D$

Kees van Boven, MD, PbD

Reinier Akkermans, MsC

Erik Bischoff, $M D, P b D$

Tim olde Hartman, MD, PbD

Department of Primary and Community Care, Radboud University Medical Center, Nijmegen, The Netherlands

\begin{abstract}
We studied the changes in presented health problems and demand for primary care since the outbreak of coronavirus disease 2019 (COVID-19) in the Netherlands. We analyzed prominent symptom features of COVID-19, and COVID-19 itself as the reason for encounter. Also, we analyzed the number and type of encounters for common important health problems. Respiratory tract symptoms related to COVID-19 were presented more often in 2020 than in 2019. We observed a dramatic increase of telephone/e-mail/Internet consultations in the months after the outbreak. Contacts for other health problems such as prevention and acute and chronic conditions plummeted substantially $(P<0.001)$; mental health problems stabilized.
\end{abstract}

Ann Fam Med 2021;19:44-47. https://doi.org/10.1370/afm.2625.

\section{INTRODUCTION}

$\mathrm{T}$ The coronavirus disease 2019 (COVID-19) pandemic in the Netherlands started at the end of February 2020. Following a steep rise in the number of cases in March 2020, the numbers decreased rapidly in the weeks following a government-forced semi-lockdown on March 12, 2020, and since then stabilized. ${ }^{1}$ In Dutch health care, family physicians (FP) play a central role: All citizens are registered with a family physician as the first point of access for health care, with specialist and hospital care only available after referral. More than $95 \%$ of care episodes are completely covered in primary care and family physicians are involved in the management of remaining episodes. ${ }^{2}$ In this context, we were interested how COVID-19 influenced daily practice. We aimed to study the changes in presented health problems and the demand for primary care during the initial crisis.

\section{METHODS}

We analyzed data of 25 family physicians in and around the city of Nijmegen, with a registered practice population of 26,225 . The demographics of Nijmegen resemble those of the Netherlands. The practices participate in the practice-based research network FaMe-net (Radboud University Medical Center, Family Medicine Network Nijmegen). ${ }^{3}$ The listed population in the network is slightly younger than the Dutch population, but comparable in sex and social class composition. ${ }^{3}$ The network has been recording routine data from family practice since 1967 . For every encounter, family physicians record the reason for encounter/presented symptoms (RFEs), duration of symptoms, diagnosis, and interventions (diagnostic testing, referral prescribed medication), under regular review of reliability of coding/classification. ${ }^{4}$ This includes the episodes of specialist care and out-of-hours services, that are reported back to the FP in almost $100 \%$ of the cases. FaMe-net classifies each condition in the

\section{Hank Schers}

Geert Grooteplein Noord 21 Nijmegen, The Netherlands henk.schers@radboudumc.nl 
Figure 1. Reasons for encounters related to COVID-19 in family practices; February-May 2019 and 2020.

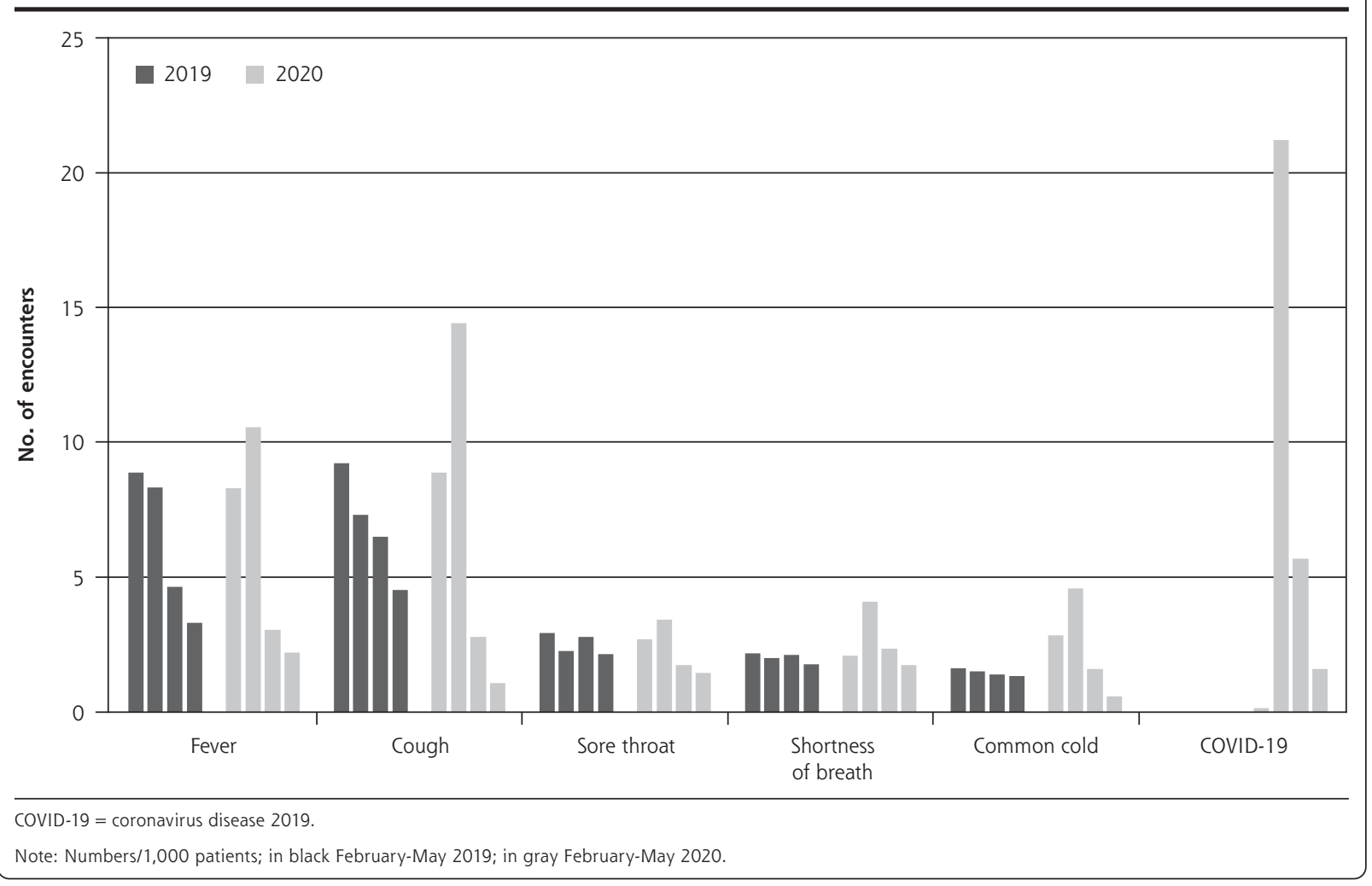

International Classification of Primary Care (ICPC) and the International Classification of Disease (ICD-10). Directly after the COVID-19 epidemic struck the Netherlands, FaMe-net started to use specific codes for COVID-19related problems (ICPC R83 and ICD-10 u.07.1 2019$\mathrm{nCoV}$ respiratory tract infection), following the ICPCmanual. ${ }^{5}$ If patients referred explicitly to COVID-19 as their reason to contact, COVID-19 was coded as the RFE. COVID-19 was coded as a diagnosis in patients: (1) with a positive polymerase chain reaction (PCR) test; or (2) with a suspicious clinical picture.

We analyzed the most prominent symptom features of COVID-19 (tiredness, cough, shortness of breath, sore throat, common cold, fever) presented in February to May 2019 and 2020, and for 2020 also COVID-19 as RFE. We also analyzed the number of practice encounters and types of encounter: practice visit (including home visit) vs telephone consultation (including e-mail/video-consultations); and encounters for a number of the most common important health problems: hypertension, diabetes mellitus, mental health problems, otitis, fatigue, and preventive counseling.

We used the Mid-P exact method to test for differences in symptom rates and encounter rates. For RFEs we tested significance for changes in distribution for
2020 compared with 2019 (correction for population size); and for different health problems the changes in encounter types with a $\chi^{2}$ test.

\section{RESULTS}

By May 31, 2020 a total of 558 COVID-19 cases were diagnosed of whom $56(10 \%)$ were confirmed by a positive PCR-test. More respiratory tract symptoms were presented in March 2020 than in March 2019 (sore throat $P=0.013$, other symptoms $P<0.001$ ), and COVID-19 became the most common respiratory tract-related reason for patients to contact the practice. From April to May 2020, presented symptoms dropped to levels lower than in 2019 (fever and cough $P<0.001$; shortness of breath $P=0.66$; common cold $P=0.20$; sore throat $P=0.002$ ) (Figure 1). The overall number of encounters with the practice remained stable (per 1,000 patients 445 in 2019, 450 in 2020; $P=0.86$ ), with an increase of telephone/e-mail/videoconsultations from $30.6 \%$ of the encounters in 2019 to $53.3 \%$ in March $2020(P<0.001)$.

From April 2019 on, hypertension, diabetes mellitus, fatigue, otitis, and preventive counseling were common reasons to contact the practice, but from early March 2020 their frequency of contact 
Figure 2. Contacts with family practices in February-May 2019 and 2020; visits and telephonele-mail consultations (numbers per 1,000 patients).
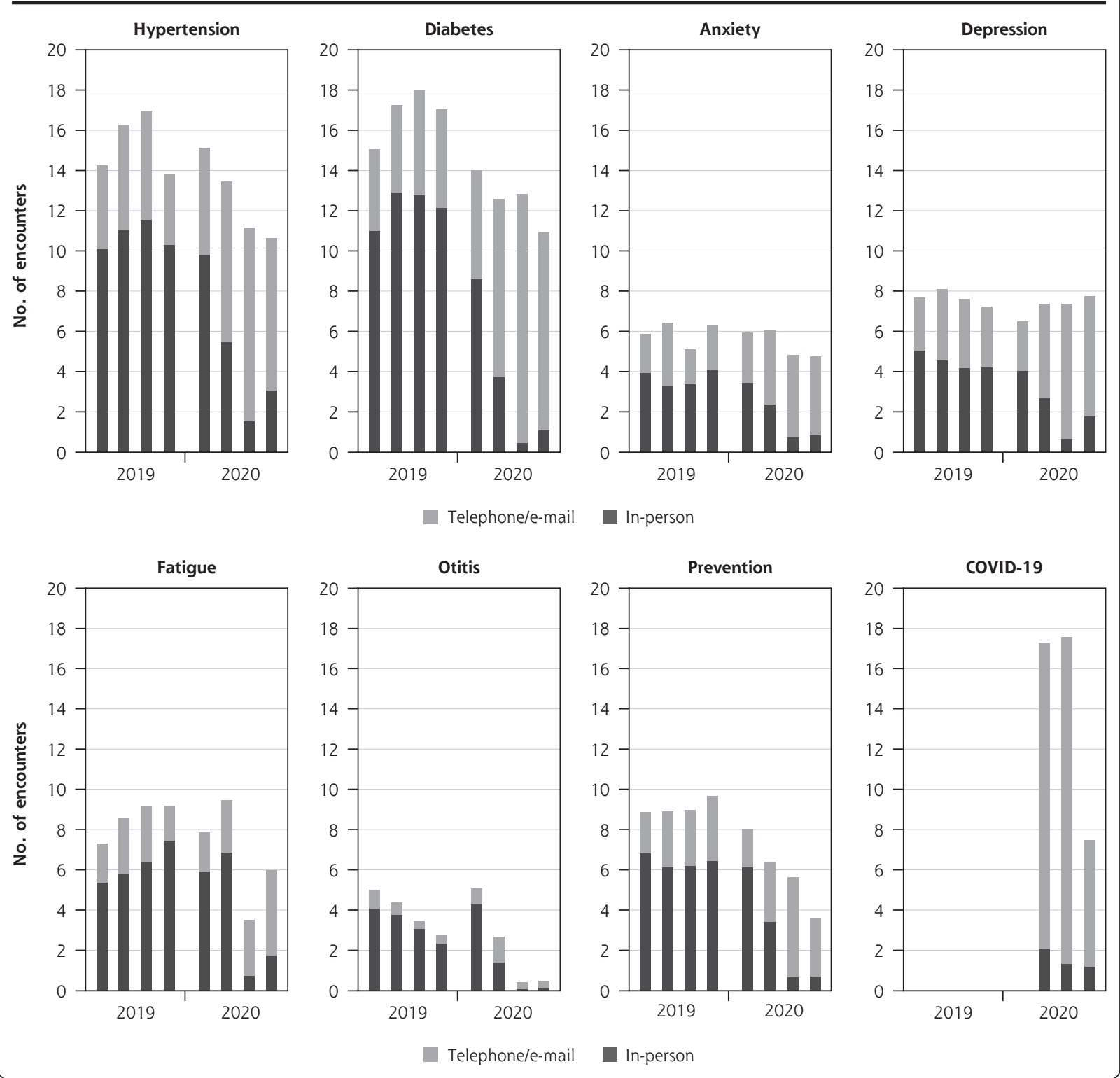

plummeted substantially and their distribution changed significantly (all $P<0.001$ ). Mental health problems were presented in comparable numbers (anxiety $P=0.040$; depression $P=0.82$ ) (Figure 2).

\section{DISCUSSION}

Our data illustrate the large impact of COVID-19 on Dutch family practice. Patients presented a large number of respiratory symptoms and COVID-19-related questions and concerns to their family physicians. This illustrates the clinical challenge to family practice. In-person encounters were replaced by distanced consultations and by telephone and e-mail. The demand for primary care changed rapidly as well: acute and chronic health problems, and prevention decreased more or less from the very moment the COVID-19 epidemic entered the country. Encounters for mental health problems stabilized.

Postponement of regular, scheduled office visits may have enabled the practice to focus on the sudden increase in COVID-19 care. But it also raised concerns of damaging effects on the health of the vulnerable: those with chronic physical and mental health problems. Our data show that, although delivered from a distance, encounters for mental health 
problems did not decrease. Encounters for preventive care dropped, however, as was the case for acute and chronic health conditions.

Our study has limitations such as it covers only 1 city in the Netherlands. At the same time, we consider this a strength because COVID-19 does not hit regions and cities simultaneously, and the study of 1 geographic region will give the best insight into the impact of COVID-19 on demand for health care and morbidity patterns.

Our data stress the importance of securing care for all health problems in primary care's preparations for a major epidemic, and avoiding the collateral damage of a health systems' single-minded focus on an epidemic.

To read or post commentaries in response to this article, see it online at https://www.AnnFamMed.org/content/19/1/44.
Key words: COVID-19; coronavirus; general practice; delivery of healthcare; primary care; the Netherlands

Submitted April 3, 2020; submitted, revised, July 28, 2020; accepted August 3, 2020.

\section{References}

1. FaMe-net. Accessed Jun 30, 2020. https://www.famenet.nl/

2. Van Weel C, Schers H, Timmermans A. Health care in the Netherlands. J Am Board Fam Med. 2012;25(Suppl 1):S12-S17.

3. Van Boven $K$, Uijen AA, van de Wiel N, et al. The diagnostic value of the patient's reason for encounter for diagnosing cancer in primary care. J Am Board Fam Med. 2017;30(6):806-812.

4. Van Weel C; Weel van C. The Continuous Morbidity Registration Nijmegen: background and history of a Dutch general practice database. Eur J Gen Pract. 2008;14(Suppl 1):5-12.

5. World Health Organization. Classifications. Accessed Jun 30, 2020. https://www.who.int/classifications/icd/en/ 